\title{
Mapping Global Research Output in Big Data during 2007-16
}

\author{
S.M.Dhawan ${ }^{\mathrm{a}}$, B.M.Gupta ${ }^{\mathrm{b}}$, Ritu Gupta ${ }^{\mathrm{c}}$ \\ ${ }^{a}$ Formerly CSIR -NPL, New Delhi (Present Address: 114 DayanandVihar, Delhi 110092). India. \\ ${ }^{b}$ Formerly CSIR -NISTADS, New Delhi (Present Address: 1173 Sector 15, Panchkula 134 113, Haryana). India. \\ ${ }^{c}$ Formerly with Sri Venkateshwara University, Tirupathi, Andhra Pradesh (Present Address: 1 K/A Arjun Nagar, Safdarjang \\ Enclave, New Delhi 110029), India.
}

\begin{abstract}
The paper examines global research in big data, as covered in Scopus database 2007-16, on a series of bibliometric indicators. The study finds that big data registered exceedingly fast growth (135.2\%), but averaged low citation impact per paper (3.75) and accounted for very low share of highly cited papers $(0.86 \%)$ in 10 years. The study reports publication trends in big data research by top countries, top institutions, top authors, top journals, major subject areas, publication modes, and country-level share of international collaborative publications. The study concludes that big data is a subject of recent origin. Given its major potential to impact business, governance, society, healthcare, industry and many other sectors, big data is fast emerging as a major discipline of interest and importance to nations, corporates, and institutions across developed and fast emerging economies.
\end{abstract}

\section{Keywords:}

Big Data Research, Global Publications; Scientometrics; Bibliometrics.

Article History:

Received: 17 May 2018

Accepted: 01 July 2018

\section{1- Introduction}

Big data refers to massive data repositories, so huge and complex in size and data types, that traditional databases find it beyond their ability to capture, manage, and process such data sets and deriving therefrom new meaning and value that can dramatically improve business policies and practices. Big data sets are growing at dazzling speed in volume and variety and processing such larger data sets using standard IT technologies is a challenge. Big data includes structured, semi-structured, and unstructured data of diverse types and sizes ranging from terabytes to zettabytes. Unstructured data includes images, email, text messages, mobile and social media updates. The essence of big data is to create order from chaos, value from volume, and fresh insights from unexplored unstructured data. Major sources contributing to big data sets include mobile and social media, internet of things, and artificial intelligence sources, and that such big data sets are swelling by the minute in volume, velocity and variety [1].

Big data is an umbrella term that refers to the use of big data analytics, big data platforms, software tools and techniques to capture, store, analyze and harness previously untapped data sources independently or together with existing enterprise data. Data analytics techniques include text analytics, machine learning, predictive analytics, data mining, statistics, and natural language processing, businesses. These techniques can make data simple, accessible, and help gain analysts deep insight into raw data and useful to make better and faster decisions; improve business processes and policies [2-3].

Over the years, big data has evolved into a research and scientific study on how to make big data work [4]. The research studies in big data are into big data technologies, big data platforms and big data infrastructure. Big data studies are also into developing user toolsets designed to examine current data streams and big data repositories, and develop low cost and low complexity big data environment that is stable, highly integrated, and scalable. The challenge in big data research studies is how to make organizations big data ready, how to make the power of big data and big data

\footnotetext{
* CONTACT: bmgupta1@gmail.com

DOI: http://dx.doi.org/10.28991/esj-2018-01135

(C) This is an open access article under the CC-BY license (https://creativecommons.org/licenses/by/4.0/).
} 
analytics available to all the parts of the organization that need them to discover valuable insights, make better decisions and solve actual business problems.

\section{1-1- Literature Review}

Of late, a number of bibliometric studies have been conducted that are national and global in scope. Amongst these studies, Halevi and Moed [4] analyzed publications data on big data from several perspectives: time line, types of published papers, geographic output, disciplinary output and thematic and conceptual development. They download data from Scopus database and in conclusion described the emergence of global big data as a research topic. Singh, Banshal, Singhal and Uddin [5] studied big data research output published during 2010-14, and as covered in both databases, Web of Knowledge and Scopus, for research growth, authorship patterns, country-level research collaboration patterns, major contributors (countries, institutions and individuals), top publication sources, thematic and emerging themes in the field. Singh and Singh [6] mapped Indian research output in the area of big data published during 2001-15, using Scopus database, for understanding current status, growth, and collaboration trends in big data research and diffusion of big data research in Indian scientific literature. Liu [7] analyzed big data research output (282 records using SSCI database during 2005-15) for understanding distribution of research by publication year, growth pattern, top journals, top subject areas, top countries/territories, academic institutions, top authors and applicability of Lotka's law. Porter, Huang, Schuehle and Youtie [8] presented a meta-analysis of big data research activity, covering 7006 research publications since 2009 from Web of Science database. Using "tech mining" (bibliometric and text analyses of research publication abstract record sets), the authors provided a research landscape of who is doing what, where, and when. Mathisen, Wienhofen and Roman [9] presented the current status of empirical research in big data by mapping the collected research (covering 1778 contributions) according to the labels: variety, volume and velocity. Besides, they identified application areas of big data. The authors concluded that the share of publications conforming to empirical results is well below the average compared to computer science research as a whole. Kalantri et al [10] analyzed 6572 papers in big data field as indexed in in Web of Science TM Core Collection database from 1980 to 19 March 2015 and reported publication trends by document type and language, year of publication, top countries, top journals, top research areas, and top authors.

There were a few other bibliometric studies but covering only application dimensions of big data field in medical science. Liao, Liao, Lee, Li, Chiclana and and Zeng [11] used visualization tools (GraphPad Prism 5, VOSviewer and CiteSpace softwares ) to identify annual trends, top authors, top journals, top institutes, country-level citations and $\mathrm{H}-$ index, keywords distribution, highly cited papers, and co-authorship status. Gua, Lia, Lia and Lianga [12] provided an overview of healthcare big data research, research hotspots and future research directions. Youtie, Porter and Hunag [13] examined a dataset of 488 social science and humanities papers written about big data and concluded that eight sub-fields are important in framing social science research about big data. The big data field covering social science is evolving from general sociological considerations towards social science applications, issues, and privacy concerns.

\section{1-2- Objectives}

The study analyses the performance of global big data research during 2007-16, based on publications, citations and international collaborative publications covered in Scopus database. The focus of study in particular was to growth characteristics and pattern of world research output and its citation received; global publications output, share and citations of top 12 most productive countries; international collaboration share of top 12 most productive countries; the subject-wise distribution of global research output and its growth and decline; identification of significant keywords; publication productivity and citation impact 100 most productive organizations and authors; leading medium of communication and characteristics of top 96 high cited papers.

\section{2- Methodology}

The global research output on big data studies was identified, retrieved and downloaded from the Scopus database (http://www.scopus.com) 2007-16, using a well formulated search strategy. The search strategy included using the term "big data" in "keyword tag", "article title tag", and "source title tag" " and restricted search output to period 2007-16 in "date range tag". This main search string was further restricted to individual country by name in "country tag" to ascertain publication output of top 12 most productive countries in big data research. The main search string was also refined by "subject area tag", "country tag", "source title tag", "journal title name" and "affiliation tag" one by one and accordingly determined distribution of big data publications output by subject, collaborating countries, author-wise, organization-wise and journal-wise, etc. For citation data, citations to publications were collected from date of publication till 27 January 2018.

(KEY ("big data") OR TITLE ("big data") OR SRCTITLE ("big data")) AND PUBYEAR > 2006 AND PUBYEAR $<2017$. 


\section{3- Analysis}

\section{3-1- Publications Distribution}

Big data research registered $136.84 \%$ CAGR growth, cumulated 26566 publications globally in 10 years during 200716, and witnessed a big jump in its annual output from just 2 in 2007 to 11104 publications in 2016. The second-half of the study period (2012-16) cumulated a five-year high of 26477 publications, compared to just 89 during the first-half (2007-11), an absolute five-year growth 29649\%. The big data research averaged 3.75 citations per paper since publication in 10 years during 2007-16, and citation impact of its five-year output dropped from 39.58 CPS in 2007-11 to $3.63 \mathrm{CPS}$ in 2012-16 (Figure 1, Table 1).

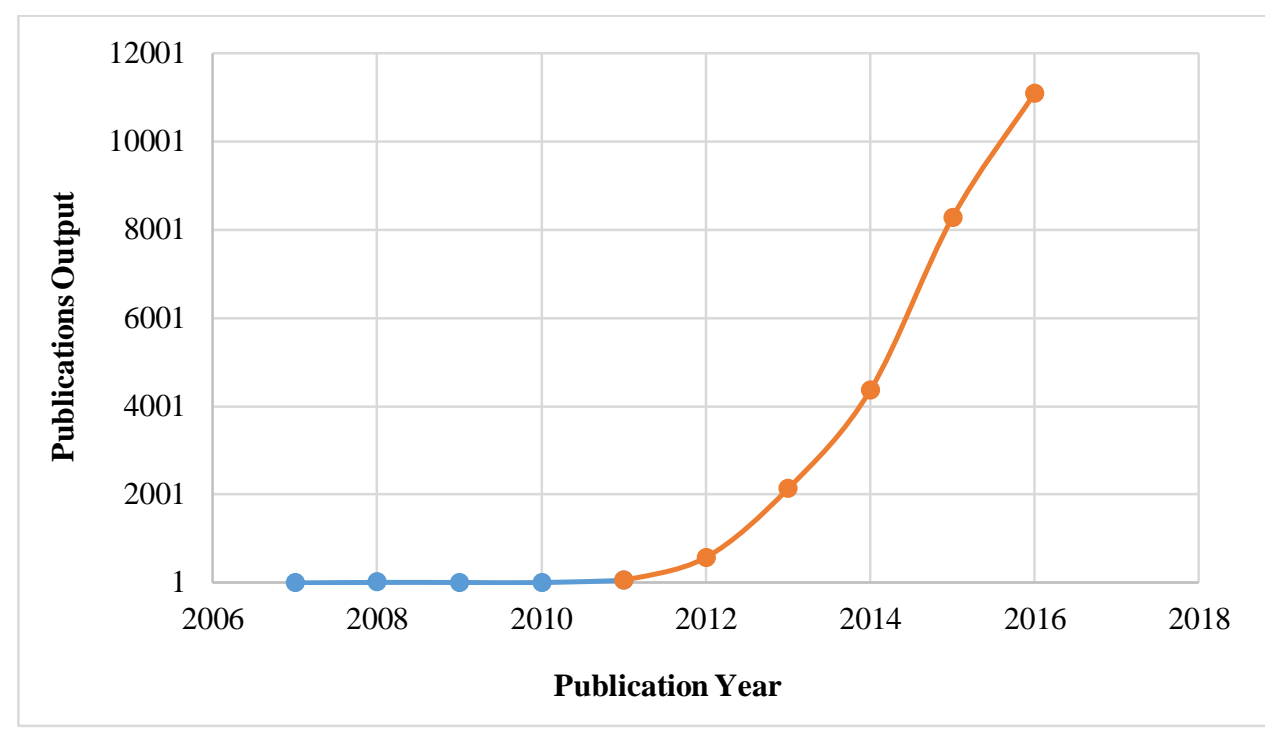

Figure 1. Global Research Output in Big Data Field: 2007-16.

Table 1. Global Research Output in Big Data Field: 2007-16.

\begin{tabular}{cccc}
\hline Year & Papers & Citations & Citations Per Paper (CPS) \\
\hline 2007 & 2 & 0 & 0 \\
2008 & 13 & 942 & 72.46 \\
2009 & 11 & 623 & 56.64 \\
2010 & 9 & 152 & 16.89 \\
2011 & 54 & 1806 & 33.44 \\
2012 & 576 & 8441 & 14.65 \\
2013 & 2138 & 18292 & 8.56 \\
2014 & 4372 & 27950 & 6.39 \\
2015 & 8287 & 28603 & 3.45 \\
2016 & 11104 & 12781 & 1.15 \\
$2007-11$ & 89 & 3523 & 39.58 \\
$2012-16$ & 26477 & 96067 & 3.63 \\
$2007-16$ & 26566 & 99590 & 3.75 \\
\hline Annual Growth & $\mathbf{2 7 5 . 8 1 \%}$ & & \\
\hline CAGR & $\mathbf{1 3 6 . 8 4 \%}$ & & \\
\hline Quinquennial Growth & $\mathbf{2 9 6 4 9 . 4 4 \%}$ & & \\
\cline { 1 - 2 } & & & \\
\hline
\end{tabular}

Bulk of big data research appeared as conference papers $(64.15 \%, 17043)$, and the remaining across other publication types such as articles $(24.18 \%, 6423)$, book chapters $(3.58 \%, 950)$, reviews $(2.64 \%, 702)$, editorials $(2.38 \%, 633)$, notes $(0.97 \%, 139)$, short surveys $(0.52 \%, 139)$, articles in press $(0.46 \%, 121)$ and the rest as conference reviews $(110)$, books (107), letters (61) and erratum(18) during 2007-16. The distribution of global publications output by language was skewed; as much as 96.65\% share (25676) appeared in English and only 2.26\% (600) in Chinese, $0.47 \%$ (125) in German, $0.21 \%$ (56) in Spanish, 0.20\% (54) in French, and the rest in 15 other languages. 


\section{3-2- Top 12 Most Productive Countries}

In all, 161 countries participated in big data research during 2007-16, but only 12 had lead the field with their cumulative global output of $92.14 \%$; their individual share varied between $2.40 \%$ and $27.98 \%$ of global output. The USA leads the world with $27.98 \%$ global share, followed closely by China (24.58\%). Both USA and China account for more than $50 \%$ of global share, followed by India (6.62\%), U.K. (5. 75\%), Germany (5.11\%), and others (Figure 2, Table 2).

Of the 12 top countries, six registered relative citation Index above the group average of 1.30: USA (1.88), UK (1.77), Canada (1.72), Australia (1.62), Italy (1.48) and Spain Korea (1.31) during the period (Table 2). China, India, Germany, and South Korea -- rated as the most productive countries after USA -- failed to register above average relative citation score, highlighting thereby gap in their performance in terms of quality and quantity of research.

Table 2. Publication Output and Global Publication Share of Top 20 Most Productive Countries in Big Data Research during 2007-16.

\begin{tabular}{|c|c|c|c|c|c|c|c|c|c|}
\hline S. No & Country Name & $\mathbf{T P}$ & TC & $\% \mathrm{TP}$ & $\% \mathrm{TC}$ & CPP & ICP & \% ICP & RCI \\
\hline 1 & USA & 7434 & 52379 & 27.98 & 52.59 & 7.05 & 776 & 10.44 & 1.88 \\
\hline 2 & China & 6529 & 21142 & 24.58 & 21.23 & 3.24 & 739 & 11.32 & 0.86 \\
\hline 3 & India & 1758 & 3298 & 6.62 & 3.31 & 1.88 & 191 & 10.86 & 0.50 \\
\hline 4 & U.K. & 1527 & 10121 & 5.75 & 10.16 & 6.63 & 733 & 48.00 & 1.77 \\
\hline 5 & Germany & 1358 & 5699 & 5.11 & 5.72 & 4.20 & 501 & 36.89 & 1.12 \\
\hline 6 & South Korea & 1071 & 3330 & 4.03 & 3.34 & 3.11 & 200 & 18.67 & 0.83 \\
\hline 7 & Australia & 922 & 5611 & 3.47 & 5.63 & 6.09 & 502 & 54.45 & 1.62 \\
\hline 8 & Japan & 904 & 2298 & 3.40 & 2.31 & 2.54 & 213 & 23.56 & 0.68 \\
\hline 9 & Italy & 825 & 4575 & 3.11 & 4.59 & 5.55 & 328 & 39.76 & 1.48 \\
\hline 10 & Canada & 818 & 5286 & 3.08 & 5.31 & 6.46 & 430 & 52.57 & 1.72 \\
\hline 11 & France & 694 & 2821 & 2.61 & 2.83 & 4.06 & 346 & 49.86 & 1.08 \\
\hline \multirow[t]{4}{*}{12} & Spain & 638 & 3123 & 2.40 & 3.14 & 4.89 & 303 & 47.49 & 1.31 \\
\hline & Total & 24478 & 119683 & & & 4.89 & 5262 & 21.50 & 1.30 \\
\hline & World Total & 26566 & 99590 & & & 3.75 & & & \\
\hline & $\begin{array}{c}\text { Share of } 12 \\
\text { countries in World } \\
\text { Total }\end{array}$ & 92.14 & & & & & & & \\
\hline
\end{tabular}

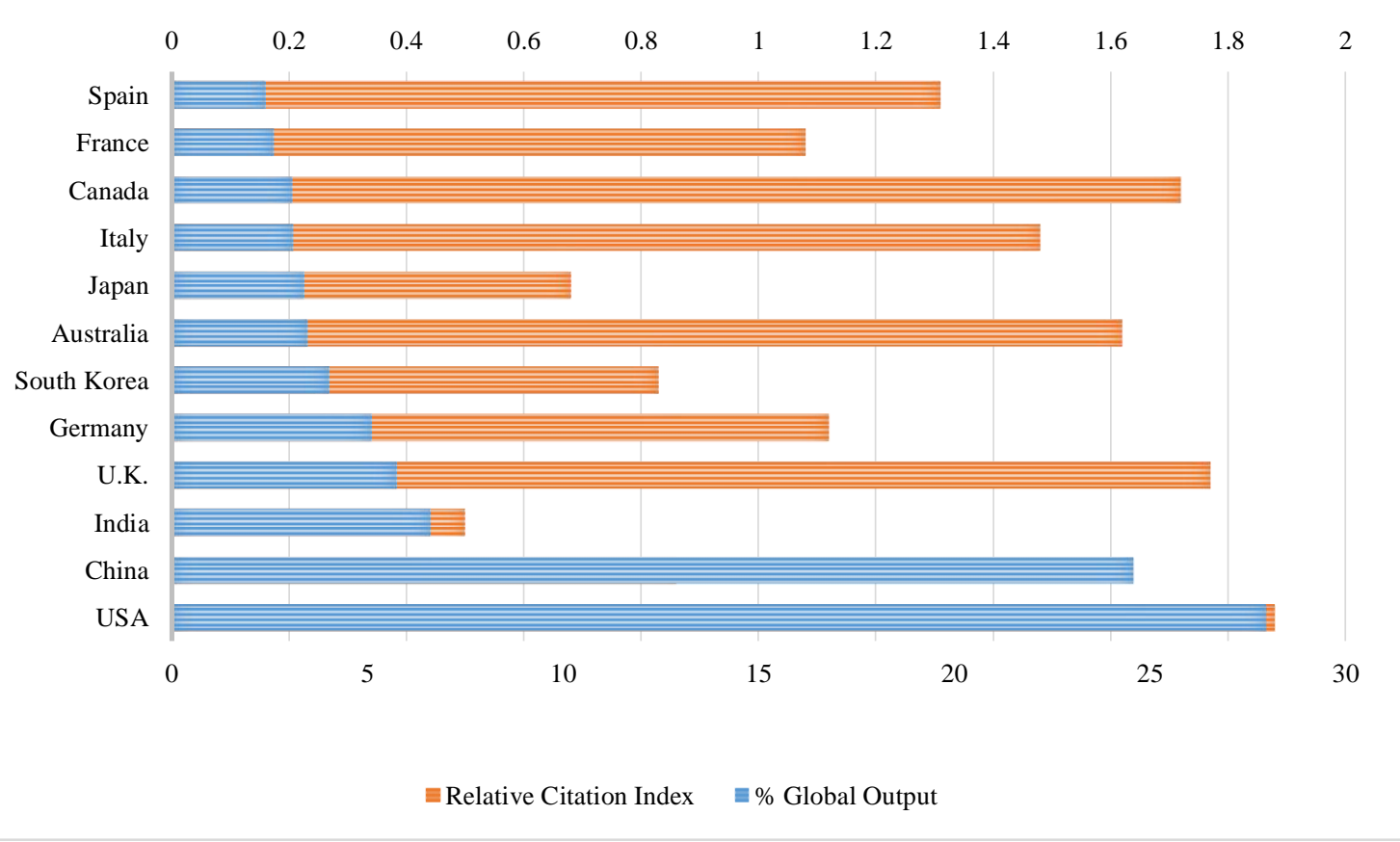

Figure 2. Quantity \& Quality Comparative Study of Top Most Productive Countries: 2007-16. 


\section{3-3- International Collaboration}

International collaboration in big data research has been common amongst top 12 most productive countries and published $10.44 \%$ to $54.45 \%$ of their national output as international collaborative publications. Australia published $54.45 \%$ of its national output, the highest amongst 12 most productive countries, as international collaborative publications, followed by Canada (52.57\%), France (49.86\%), France (45.69\%), U.K. (48.0\%), Spain (47.49\%), Italy $(39.76 \%)$, Germany $(36.89 \%)$, Japan $(23.56 \%)$, South Korea (18.67\%), China (11.32\%), India (10.86\%) and USA (10.44\%) during 2007-16.

\section{3-4- Subject-Wise Distribution of Research Output}

The global big data research cuts across several disciplines as reflected in Scopus database classification. Computer science is the most studied subject in big data research accounting for $67.99 \%$ subject share, the highest compared to other subjects, followed by engineering $(42.65 \%)$, social sciences $(13.80 \%)$, and the rest in other subjects as covered in Table 3.

Table 3. Subject-Wise Break-up of Global Publications on Mobile Research during 2007-16.

\begin{tabular}{|c|c|c|c|c|c|c|c|}
\hline \multirow{2}{*}{ S.No } & \multirow{2}{*}{ Subjects* } & \multicolumn{3}{|c|}{ Number of Papers (TP) } & \multicolumn{2}{|c|}{ Activity Index } & \multirow{2}{*}{$\% \mathrm{TP}$} \\
\hline & & 2007-11 & 2012-16 & $2007-16$ & 2007-11 & 2012-16 & \\
\hline 1 & Computer Science & 47524 & 47489 & 95013 & 103.43 & 96.79 & 67.69 \\
\hline 2 & Engineering & 32072 & 27791 & 59863 & 110.78 & 89.90 & 42.65 \\
\hline 3 & Social Sciences & 11856 & 7511 & 19367 & 126.58 & 75.10 & 13.80 \\
\hline 4 & Mathematics & 5172 & 6893 & 12065 & 88.64 & 110.64 & 8.59 \\
\hline 5 & Medicine & 2532 & 5149 & 7681 & 68.16 & 129.82 & 5.47 \\
\hline 6 & Physics \& Astronomy & 1682 & 2893 & 4575 & 76.02 & 122.46 & 3.26 \\
\hline 7 & $\begin{array}{c}\text { Business, Accounting \& } \\
\text { Management }\end{array}$ & 1905 & 2112 & 4017 & 98.06 & 101.82 & 2.86 \\
\hline 8 & $\begin{array}{l}\text { Biochemistry, Genetics \& } \\
\text { Molecular Biology }\end{array}$ & 1516 & 1622 & 3138 & 99.90 & 100.10 & 2.24 \\
\hline 9 & Materials Science & 1349 & 1765 & 3114 & 89.58 & 109.76 & 2.22 \\
\hline \multirow[t]{2}{*}{10} & Decision Science & 939 & 1186 & 2125 & 91.37 & 108.08 & 1.51 \\
\hline & World Total & 67887 & 72488 & 140375 & & & \\
\hline
\end{tabular}

*There was overlapping of research output across various subjects

\section{4-6- Significant Keywords}

Around 69 significant keywords were identified from the literature that seeks to highlight broad trends in big data research. These keywords are listed in Table 4 in the decreasing order of their occurrence during 2007-16.

Table 4. List of Significant Keywords appearing in Big Data Research Literature during 2007-16.

\begin{tabular}{|c|c|c|c|c|c|}
\hline S.No & Name of Keyword & Frequency & S.No & Name of Keyword & Frequency \\
\hline 1 & Big data & 21961 & 36 & Ubiquitous computing & 519 \\
\hline 2 & Data mining & 3733 & 37 & Big data analytics & 487 \\
\hline 3 & Data handling & 2864 & 38 & Metadata & 484 \\
\hline 4 & Digital Storage & 2454 & 39 & Commerce & 463 \\
\hline 5 & Cloud computing & 2202 & 40 & Bioinformatics & 447 \\
\hline 6 & Algorithms & 2126 & 41 & Computer architecture & 446 \\
\hline 7 & Artificial intelligence & 2107 & 42 & Websites & 434 \\
\hline 8 & Information management & 2076 & 43 & Benchmarking & 441 \\
\hline 9 & Distributed compute systems & 1876 & 44 & Network security & 426 \\
\hline 10 & Social networking (Online) & 1679 & 45 & Information processing & 425 \\
\hline 11 & Learning systems & 1585 & 46 & Information retrieval & 425 \\
\hline 12 & Internet & 1513 & 47 & Health care & 419 \\
\hline 13 & Map reduce & 1260 & 48 & World Wide Web & 406 \\
\hline 14 & Big datum & 1230 & 49 & Behavioral research & 402 \\
\hline 15 & Hadoop & 980 & 50 & Natural language processing & 402 \\
\hline 16 & Semantics & 945 & 51 & Security of Data & 396 \\
\hline 17 & Data privacy & 916 & 52 & Computer software & 395 \\
\hline 18 & Internet of things & 899 & 53 & Data reduction & 393 \\
\hline 19 & Classification (of information) & 832 & 54 & Iterative methods & 393 \\
\hline
\end{tabular}




\begin{tabular}{|c|c|c|c|c|c|}
\hline S.No & Name of Keyword & Frequency & S.No & Name of Keyword & Frequency \\
\hline 20 & Optimization & 814 & 55 & Web services & 384 \\
\hline 21 & Information analysis & 808 & 56 & Intelligent systems & 381 \\
\hline 22 & Forecasting & 769 & 57 & Semantic web & 381 \\
\hline 23 & Data visualization & 739 & 58 & Computational linguistics & 378 \\
\hline 24 & Complex networks & 732 & 59 & Image processing & 364 \\
\hline 25 & Cluster algorithms & 722 & 60 & Software engineering & 362 \\
\hline 26 & Machine learning & 686 & 61 & Scalability & 360 \\
\hline 27 & Information systems & 672 & 62 & Application programming & 359 \\
\hline 28 & Database systems & 660 & 63 & Neural networks & 360 \\
\hline 29 & Search engines & 610 & 64 & Cryptography & 337 \\
\hline 30 & Query processing & 580 & 65 & Transportation & 316 \\
\hline 31 & Big data applications & 572 & 65 & Mobile devices & 313 \\
\hline 32 & Learning algorithms & 572 & 66 & Pattern recognition & 293 \\
\hline 33 & Computation theory & 556 & 67 & Virtual reality & 293 \\
\hline 34 & Social media & 543 & 68 & Embedded systems & 291 \\
\hline 35 & Education & 529 & 69 & Data communication systems & 285 \\
\hline
\end{tabular}

\section{4-7- Top 100 Organizations in Big Data Research}

Top 100 most productive organizations originated from 15 countries, and they contributed 60 to 347 publications each, accounted for 36.01\% (9566) global publications share and 61.33\% (61074) global citations share during 200716. Of 100 organizations, 70 had originated from just two countries, 36 from USA (with 3232 papers) and 34 from China (3801 papers). The rest originated from 13 countries, 7 of which were from Australia (613 papers), 4 from Hong Kong (275 papers), and others. Singapore registered the highest impact (14.23), the highest h-index (15.50), and Hong Kong the highest international collaborative publications (69.82\% of national output). (Figure 3, Table 5)

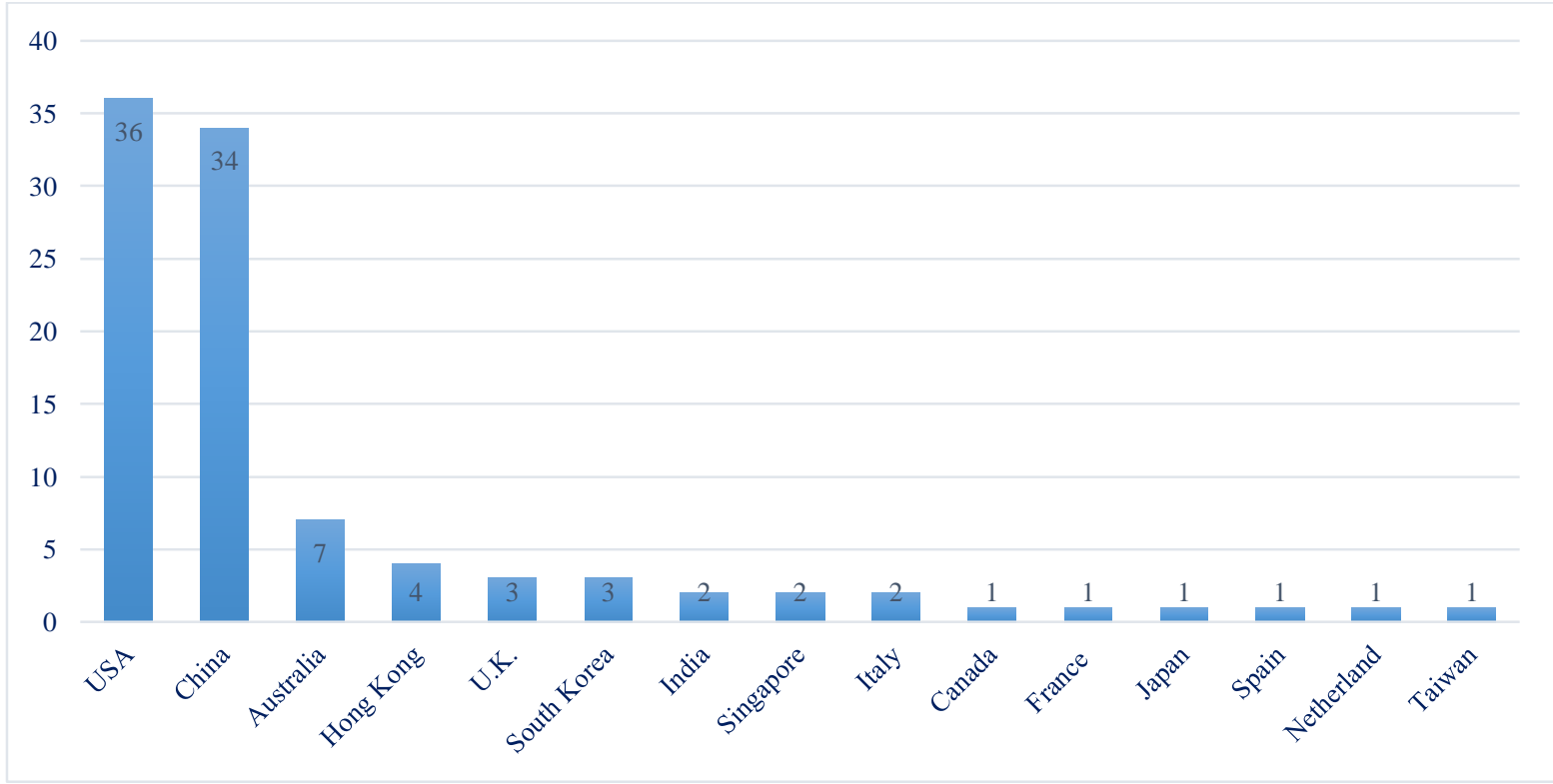

Figure 3. Top 100 Organizations in Big Data Research by Country of Origin: 2007-16.

Table 5. Publication and Citation Profile of Top 100 Organizations by Country of Origin: 2007-16.

\begin{tabular}{cccccccccc}
\hline S. No. & Country Name & $\begin{array}{c}\text { Organizations } \\
\text { Count }\end{array}$ & TP & TC & CPP & HI & ICP & \%ICP \\
\hline 1 & USA & 36 & 3232 & 27493 & 8.51 & 12.56 & 1054 & 32.61 \\
2 & China & 34 & 3801 & 16736 & 4.40 & 9.18 & 999 & 26.28 \\
3 & Australia & 7 & 613 & 4349 & 7.09 & 12.86 & 341 & 55.63 \\
4 & Hong Kong & 4 & 275 & 1898 & 6.90 & 10.50 & 192 & 69.82 \\
5 & U.K. & 3 & 266 & 2898 & 10.89 & 15.00 & 151 & 56.77 \\
6 & South Korea & 3 & 220 & 865 & 3.93 & 7.0 & 46 & 20.91 \\
7 & India & 2 & 205 & 306 & 1.49 & 6.00 & 17 & 8.29
\end{tabular}




\begin{tabular}{ccccccccc}
\hline S. No. & Country Name & $\begin{array}{c}\text { Organizations } \\
\text { Count }\end{array}$ & TP & TC & CPP & HI & ICP & \% ICP \\
\hline 8 & Singapore & 2 & 191 & 2717 & 14.23 & 15.50 & 128 & 67.02 \\
9 & Italy & 2 & 152 & 800 & 5.26 & 10.00 & 61 & 40.13 \\
10 & Canada & 1 & 98 & 1028 & 10.49 & 14.00 & 69 & 70.41 \\
11 & France & 1 & 139 & 499 & 3.59 & 12.00 & 49 & 35.25 \\
12 & Japan & 1 & 86 & 160 & 1.86 & 6.00 & 17 & 19.77 \\
13 & Spain & 1 & 77 & 439 & 5.70 & 10.00 & 47 & 61.04 \\
14 & Netherland & 1 & 76 & 260 & 3.42 & 9.00 & 35 \\
15 & Taiwan & 1 & 70 & 412 & 5.89 & 11.00 & 24 & 36.05 \\
& Total & $\mathbf{1 0 0}$ & $\mathbf{9 5 6 6}$ & $\mathbf{6 1 0 7 4}$ & $\mathbf{6 . 3 8}$ & $\mathbf{1 0 . 9 5}$ & $\mathbf{3 3 . 9 7}$ \\
\hline
\end{tabular}

Big data research involved participation of a total of 6802 global organizations, of which 5137 contributed 1-5 papers each, 766 organizations 6-10 papers each, 310 organizations 11-20 papers each, 230 organizations 21-40 papers each, 124 organizations 41-100 papers each and 235 organizations 101-348 papers each. On further analyzing these 100 organizations, it was observed that:

- Of the top 100 organizations, 38 registered productivity above the group average of 95.66 publications per organization: Tsinghua University ,China (347 papers), Beijing University of Posts and Telecommunications, China (220 papers), Ministry of Education, China (209 papers), Shanghai Jiao Tong University, China (182 papers), Wuhan University, China (163 papers), National University of Defense Technology, China (161 papers), Carnegie Mellon University, USA (142 papers), Massachusetts Institute of Technology, USA (139 papers), CNRS Centre National de la Recherche Scientifique, France (139 papers), Beihang University, China (136 papers), Peking University, China (136 papers), IBM Thomas J. Watson Research Center, USA (132 papers), etc. Among the 38 organizations, 15 each were from USA and China, 3 from Australia, 1 each from Canada, France, India, Singapore and U.K. The scientific profile of top 20 most productive organizations are shown in Table 6

- Of the top 100 organizations, 41 posted citation impact above group average of 6.38 citations per publication: University of Arizona, USA (19.58), University of California, Berkeley, USA (17.17), Nanyang Technological University, Singapore (17.14), Georgia State University, USA (15.98), Stanford University, USA (15.81), Microsoft Research, USA (13.90), University of California, San Diago, USA (12.79), Google, Inc., USA (12.58), Institute of Computing Technology, CAS, China (12.53), University of Oxford, U.K. (11.73), University of Science and Technology of China (11.54), University College London, U.K. (11.19), MIT, USA (11.06), University of Southern California, USA (10.08), University of Toronto, Canada (10.49), National University of Singapore (10.43), etc. Among the 41 organizations, 21 were from USA, 7 from China, 4 from Australia, 3 each from U.K. and Hong Kong, 2 from Singapore and 1 from Canada. The scientific profile of top 20 most productive organizations are shown in Table 6.

- Of the top 100 organizations, 41 contributed international collaborative publications (ICP) above the group average share (33.97\%): City University of Hong Kong (74.29\%), National University of Singapore (73.49\%), Hong Kong Polytechnic University (72.0\%), University of Toronto, Canada (70.41\%), Chinese University of Hong Kong (69.57\%), Deakin University, Australia (68.57\%), University of Oxford, U.K. (67.59\%), University of Technology, Sydney, Australia (62.83\%), Hong Kong University of Science and Technology (62.30\%), Nanyang Technological University, Singapore (62.04\%), etc. Of the 49 organizations, 19 were from USA, 7 from Australia, 6 from China, 4 from Hong Kong, 3 from U.K., 2 each from Italy and Singapore, and 1 each from Canada, Netherlands, South Korea, Spain and Taiwan. The scientific profile of top 20 most productive organizations are shown in Table 6.

- Of the top 100 organizations, 41 registered relative citation index (RCI) above the group average (1.70): University of Arizona, USA (5.22), University of California, Berkeley, USA (4.58), Nanyang Technological University, Singapore (4.57), Georgia State University, USA (4.26), Stanford University, USA (4.22), Microsoft Research, USA (3.71), University of California, San Diego, USA (3.41), Google, Inc., USA (3.35), Institute of Computing Technology, China (3.34), University of Oxford, U.K. (3.13), University of Science and Technology of China (3.08), University College London, U.K. (2.98), etc. Of the 41 organizations, 21 were from USA, 7 from Canada, 4 from Australia, 3 each from U.K. and Hong Kong, 2 from Singapore and 1 from Canada. The scientific profile of top 20 most productive organizations are shown in Table 7. 
Table 6. Scientometric Profile of Top 20 Global Organizations in Big Data Research by Productivity: 2007-16.

\begin{tabular}{lcccccccc}
\hline S.No & Name of the Organization & TP & TC & CPP & HI & ICP & \% ICP & RCI \\
\hline 1 & Tsinghua University & 347 & 2453 & 7.07 & 20 & 110 & 31.70 & 3 \\
2 & Beijing University of Posts and Telecommunications & 220 & 749 & 3.40 & 10 & 36 & 16.36 & 1 \\
3 & Ministry of Education China & 209 & 772 & 3.69 & 14 & 34 & 16.27 & 0 \\
4 & Shanghai Jiao Tong University & 182 & 1271 & 6.98 & 14 & 65 & 35.71 & 1 \\
5 & Wuhan University & 163 & 556 & 3.41 & 12 & 34 & 20.86 & 0 \\
6 & National University of Defense Technology & 161 & 456 & 2.83 & 11 & 28 & 17.39 & 1 \\
7 & Carnegie Mellon University & 142 & 1289 & 9.08 & 17 & 53 & 37.32 & 2 \\
8 & Massachusetts Institute of Technology & 139 & 1538 & 11.06 & 21 & 57 & 41.01 & 2 \\
9 & CNRS Centre National de la Recherche Scientifique & 139 & 499 & 3.59 & 12 & 49 & 35.25 & 0 \\
10 & Beihang University & 136 & 454 & 3.34 & 9 & 39 & 28.68 & 0 \\
11 & Peking University & 136 & 363 & 2.67 & 11 & 33 & 24.26 & 0 \\
12 & IBM Thomas J. Watson Research Center & 132 & 1337 & 10.13 & 17 & 52 & 39.39 & 1 \\
13 & Huazhong University of Science and Technology & 131 & 1218 & 9.30 & 13 & 53 & 40.46 & 1 \\
14 & University of Southern California & 129 & 1378 & 10.68 & 17 & 40 & 31.01 & 2 \\
15 & Zhejiang University & 125 & 516 & 4.13 & 11 & 38 & 30.40 & 0 \\
16 & Amity Univerity, Noida & 124 & 117 & 0.94 & 4 & 10 & 8.06 & 0 \\
17 & Harbin Institute of Technology & 118 & 270 & 2.29 & 7 & 30 & 25.42 & 0 \\
18 & Georgia Institute of Technology & 114 & 661 & 5.80 & 14 & 35 & 30.70 & 0 \\
19 & University of Technology Sydney & 113 & 975 & 8.63 & 18 & 71 & 62.83 & 0 \\
20 & Nanjing University & 113 & 592 & 5.24 & 15 & 37 & 32.74 & 0 \\
\hline
\end{tabular}

Table 7. Relative Citation Index: of Top 20 Organizations in Big Data Research by 2007-16.

\begin{tabular}{|c|c|c|c|c|c|c|c|c|}
\hline S.No & Name of the Organization & TP & TC & CPP & HI & ICP & $\%$ ICP & RCI \\
\hline 1 & University of Arizona & 78 & 1527 & 19.58 & 12 & 28 & 35.90 & 5.22 \\
\hline 2 & UC Berkeley & 104 & 1786 & 17.17 & 20 & 39 & 37.50 & 4.58 \\
\hline 3 & Nanyang Technological University & 108 & 1851 & 17.14 & 18 & 67 & 62.04 & 4.57 \\
\hline 4 & Georgia State University & 83 & 1326 & 15.98 & 7 & 34 & 40.96 & 4.26 \\
\hline 5 & Stanford University & 89 & 1407 & 15.81 & 16 & 29 & 32.58 & 4.22 \\
\hline 6 & Microsoft Research & 63 & 876 & 13.90 & 14 & 28 & 44.44 & 3.71 \\
\hline 7 & University of California, San Diego & 86 & 1100 & 12.79 & 16 & 26 & 30.23 & 3.41 \\
\hline 8 & Google Inc. & 69 & 868 & 12.58 & 15 & 24 & 34.78 & 3.35 \\
\hline 9 & $\begin{array}{c}\text { Institute of Computing Technology Chinese } \\
\text { Academy of Sciences }\end{array}$ & 92 & 1153 & 12.53 & 13 & 24 & 26.09 & 3.34 \\
\hline 10 & University of Oxford & 108 & 1267 & 11.73 & 19 & 73 & 67.59 & 3.13 \\
\hline 11 & University of Science and Technology of China & 97 & 1119 & 11.54 & 10 & 31 & 31.96 & 3.08 \\
\hline 12 & University College London & 70 & 783 & 11.19 & 12 & 34 & 48.57 & 2.98 \\
\hline 13 & Massachusetts Institute of Technology & 139 & 1538 & 11.06 & 21 & 57 & 41.01 & 2.95 \\
\hline 14 & University of Southern California & 129 & 1378 & 10.68 & 17 & 40 & 31.01 & 2.85 \\
\hline 15 & University of Toronto & 98 & 1028 & 10.49 & 14 & 69 & 70.41 & 2.80 \\
\hline 16 & National University of Singapore & 83 & 866 & 10.43 & 13 & 61 & 73.49 & 2.78 \\
\hline 17 & IBM Thomas J. Watson Research Center & 132 & 1337 & 10.13 & 17 & 52 & 39.39 & 2.70 \\
\hline 18 & Microsoft Corporation & 66 & 637 & 9.65 & 12 & 24 & 36.36 & 2.57 \\
\hline 19 & Imperial College London & 88 & 848 & 9.64 & 14 & 44 & 50.00 & 2.57 \\
\hline 20 & Huazhong University of Science and Technology & 131 & 1218 & 9.30 & 13 & 53 & 40.46 & 2.48 \\
\hline
\end{tabular}

Top 100 organizations in big data research posted constant decline in publications productivity, but top 40 posted declines faster than the rest 60s. Top 100 organizations revealed inconsistent trend in citations impact. Bottom 60 organizations (associated with lower productivity) posted higher citations per paper compared to top 40s (associated with higher productivity). This implies that top 40 organizations differ in terms of quality and quantity of research far more than the rest 60s. Furthermore, the productivity data analysis reveals that top 10 amongst 100 organizations lead in publications productivity (Figure 4). 


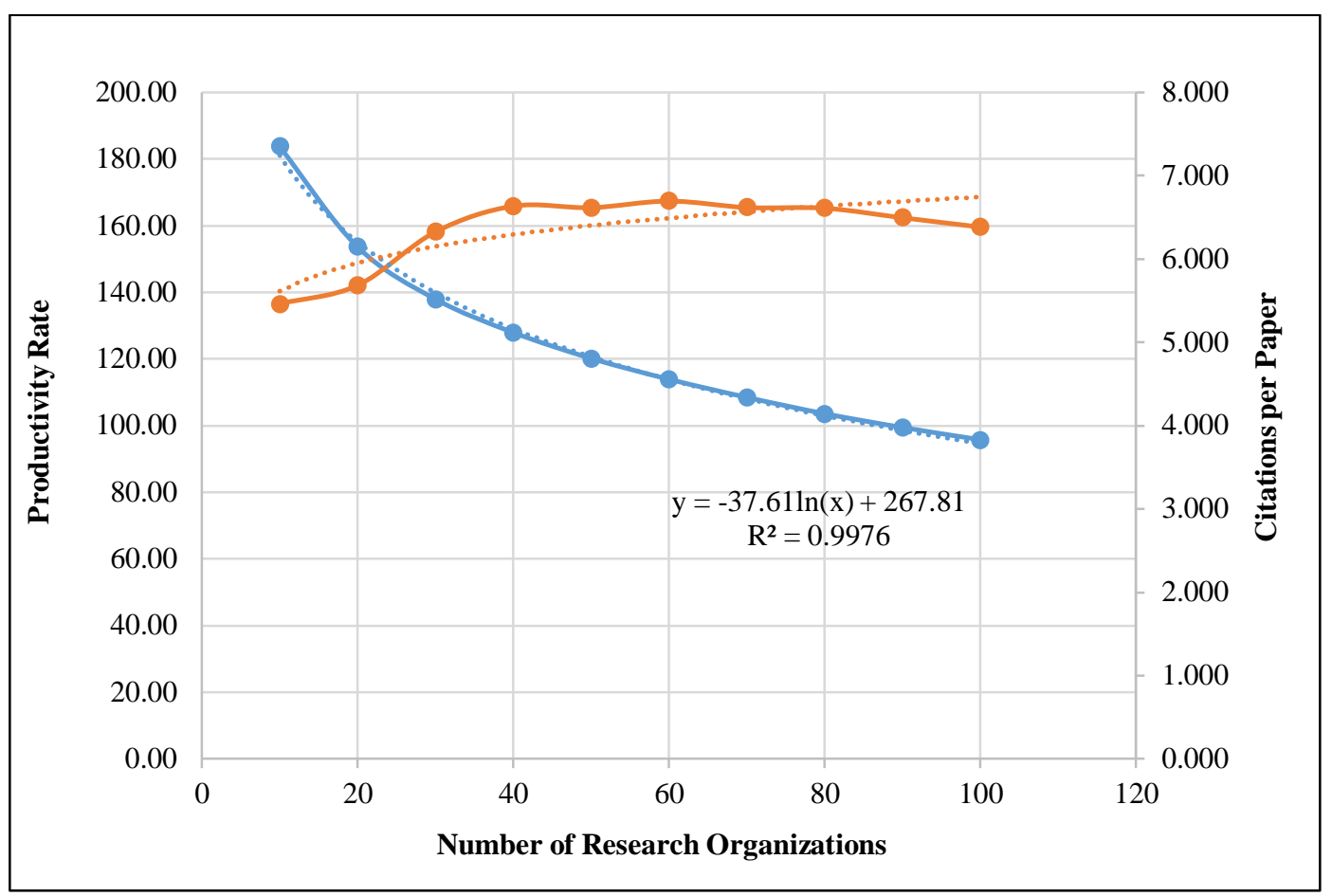

Figure 4. Top 100 Organizations Big Data Research: Quantity vs Quality Performance.

\section{3-8- Top 100 Most Productive Authors in Big Data Research}

A total of 9269 authors participated in big data research during 2007-16, of which 8452 contributed 1-5 papers each, 621 authors 6-10 papers each, 174 authors 11-20 papers each and 22 authors 21-47 papers each.

Top 100 most productive authors in big data research varied in their productivity from 13 to 47 publications in 10 years. Of these 100 organizations, 24 were from USA (with 422 papers, 19 from China ( 3410 papers), 12 from Australia (257 papers), 6 each from Italy and U.K. (121 and 99 papers), and others. In terms of citation impact per paper, Sweden registered the highest impact (18.60), followed by Italy (12.59), Australia (12.41) and others (Table 8).

Table 8. Publication and Citation Profile of Top 100 Authors by Country of Origin: 2007-16.

\begin{tabular}{cccccccc}
\hline S. No. & Country Name & $\begin{array}{c}\text { No. of } \\
\text { Authors }\end{array}$ & TP & TC & CPP & HI & \% ICP \\
\hline 1 & USA & 24 & 422 & 3908 & 9.26 & 5.58 & 31.52 \\
2 & China & 19 & 3410 & 2496 & 7.32 & 5.26 & 31.38 \\
3 & Australia & 12 & 257 & 3190 & 12.41 & 8.25 & 69.65 \\
4 & Italy & 6 & 121 & 778 & 6.43 & 5.0 & 24.79 \\
5 & U.K. & 6 & 99 & 657 & 6.64 & 5.67 & 60.61 \\
6 & South Korea & 5 & 81 & 545 & 6.73 & 5.20 & 43.21 \\
7 & Germany & 4 & 66 & 504 & 7.64 & 5.25 & 22.73 \\
8 & Canada & 4 & 67 & 658 & 9.82 & 5.75 & 71.64 \\
9 & Italy & 3 & 58 & 730 & 12.59 & 7.33 & 72.41 \\
10 & Japan & 3 & 50 & 172 & 3.44 & 4.0 & 66.0 \\
11 & Austria & 2 & 31 & 369 & 11.90 & 6.50 & 70.97 \\
12 & Romania & 2 & 29 & 191 & 6.59 & 4.50 & 27.59 \\
13 & Norway & 1 & 22 & 70 & 3.18 & 4.0 & 54.55 \\
14 & Portugal & 1 & 15 & 53 & 3.53 & 5.0 & 6.67 \\
15 & Macau & 1 & 13 & 74 & 5.69 & 5.0 & 53.85 \\
16 & Netherlands & 1 & 114 & 82 & 5.86 & 5.0 & 50.0 \\
17 & France & 1 & 13 & 70 & 5.38 & 4.0 & 76.92 \\
18 & Switzerland & 1 & 15 & 61 & 4.07 & 4.0 & 73.33 \\
19 & Taiwan & 1 & 13 & 32 & 2.46 & 2.0 & 46.15 \\
20 & Sweden & 1 & 15 & 279 & 18.60 & 10.0 & 100.0 \\
21 & Denmark & 1 & 14 & 55 & 3.93 & 5.0 & 92.86 \\
22 & India & 1 & 14 & 44 & 3.14 & 5.0 & 0.00 \\
\hline
\end{tabular}

Together these 100 authors accounted for $6.70 \%$ (1779 publications) global share and 15.08\% (15018) global citation share during 2007-16. On further analysis of these 100 authors, it was observed that:

- Thirty five authors productivity among top 100 authors in big data varied from 60 to 347 publications cumulated in 10 years: A. Cuzzocrea (Italy)(47), R. Ranjan (Australia)(41), J. Chen (Australia)(34), X. Lu (USA) and G. Yu (China)(29 each), A. Y. Zomaya (Australia)(27), X. Zhang (U.K.) and L. T. Yang (China)(25 each), S. Nepal 
(Australia)(24), F. Herrera (Spain)(23), etc. Of these 35 authors, 10 were from USA, 7 from China, 5 from Australia, 2 each from Japan, South Korea, Spain and U.K. and 1 each from Canada, Germany, Italy, Norway and Switzerland (Table 9)

- Thirty six (with citation impact ranging from 0.14 to 54.05) amongst 100 authors registered citation impact above group average of 8.44 citations per publication: H. Chen (USA)(54.05), X. Meng (Romania)(20.0), F. Herrera (Spain)(19.52), J. Wan (China)(19.33), C. Yang (Spain)(19.33), A. V. Vasilakos (Sweden)(18.60), C. Liu (Spain)(18.06), T. Rabl (Canada)(17.10), L. Wang(China)(16.52), A. Y. Zomaya (Australia)(16.19), X. Zhang (USA)(15.76), Z. Xu (China)(15.67), V.Markl (Germany)(15.44), R. Ranjan (Australia)(15.32), M. Poess (USA)(15.27), etc. during 2007-16. Of these 36 authors, 10 were from USA, 6 from Australia, 5 from China, 4 from Spain, 3 from U.K., 2 each from Austria and Canada, and 1 each from Germany, Italy, Romania and Sweden.

- Forty five (with ICP ranging from $0.0 \%$ to $100.00 \%$ ) amongst 100 authors accounted for international collaborative publications (ICP) share above the group average (44.86\%): A.Y. Zomaya (Australia), A.V. Vasilakos (Sweden), E. Kharlamov (U.K.), I. Horrocks (U.K.) and K. Li (USA)(100\% EACH), R. Vatrapu (Denmark)(92.86\%), L.T. Yang (China)(92.0\%), T. Rabl(Canada)(90.0\%) and Q. Jin (Japan)(90.0\% each), S. Yu (Australia)(88.89\%), J. Ma (Canada)(87.50\%), F. Xhafa (Spain)(81.82\%), etc. Of the 45 authors, 9 were from Australia, 8 from USA, 4 from China, 3 each from Canada and U.K., 2 each from Austria and Taiwan, and 1 each from France, Italy, Macau, Netherland, Norway, South Korea and Sweden.

- Thirty six (with RCI ranging from 0.04 to 14.41 ) amongst 100 top authors accounted for relative citation index (RCI) share above the group average (2.25): H. Chen (USA)(14.41), X. Meng (Romania)(5.33), F. Herrera (Spain)(5.21), C. Yang (Australia)(5.16), J. Wan (China)(5.16), A. V. Vasilakos (Sweden)( 4.96), C.Liu (Spain)(4.81), T. Rabl (Canada)(4.56), L. Wang (China)(4.41), A.Y. Zomaya (Australia)(4.32), X. Zhang (USA)(4.20), Z. Xu (China)(4.18), V. Markl (Gerany)(4.12), R. Ranjan (Australia)(4.08), M. Poess (USA)(4.07), etc. during 2007-16. Of these 36 authors, 11 were from USA, 4 from Australia, 4 from China, 3 each from Span and U.K. , 2 from Austria and Canada, 1 each from Germany, Italy and Romania (Table 10).

Table 9. Top 20 Most Productive Global Authors in Big Data Research, 2007-16.

\begin{tabular}{|c|c|c|c|c|c|c|c|c|c|}
\hline S.No & $\begin{array}{l}\text { Name of the } \\
\text { Author }\end{array}$ & Affiliation of the Author & TP & $\mathbf{T C}$ & CPP & HI & ICP & $\% \mathrm{ICP}$ & RCI \\
\hline 1 & A. Cuzzocrea & $\begin{array}{l}\text { ICAR-CNR University of Calabria, } \\
\text { Italy }\end{array}$ & 47 & 408 & 8.68 & 7 & 23 & 48.94 & 2.31 \\
\hline 2 & R.Ranjan & $\begin{array}{c}\text { Commonwealth Scientific \& } \\
\text { Industrial Organization, Australia }\end{array}$ & 41 & 628 & 15.32 & 15 & 31 & 75.61 & 4.08 \\
\hline 3 & J.Chen & $\begin{array}{c}\text { University of Technology Sydney, } \\
\text { Australia }\end{array}$ & 34 & 473 & 13.91 & 12 & 23 & 67.65 & 3.71 \\
\hline 4 & X.Lu & Ohio State University, USA & 29 & 197 & 6.79 & 8 & 7 & 24.14 & 1.81 \\
\hline 5 & G.Yu & Northeastern University, China & 29 & 108 & 3.72 & 5 & 6 & 20.69 & 0.99 \\
\hline 6 & A.Y.Zomaya & University of Sydney, Australia & 27 & 437 & 16.19 & 11 & 30 & 111.11 & 4.32 \\
\hline 7 & L.T.Yang & $\begin{array}{c}\text { Huazhong University of Science \& } \\
\text { Technology, China }\end{array}$ & 25 & 173 & 6.92 & 7 & 23 & 92.00 & 1.85 \\
\hline 8 & X.Zhang & University of Manitobo, Canada & 25 & 394 & 15.76 & 12 & 17 & 68.00 & 4.20 \\
\hline 9 & S.Nepal & University of Dereby, U.K. & 24 & 201 & 8.38 & 8 & 7 & 29.17 & 2.23 \\
\hline 10 & L.Wang & $\begin{array}{c}\text { Institute of Remote Sensing \& Digital } \\
\text { Earth, China }\end{array}$ & 23 & 380 & 16.52 & 13 & 18 & 78.26 & 4.41 \\
\hline 11 & F.Herrera & University of Granada, Spain & 23 & 449 & 19.52 & 10 & 14 & 60.87 & 5.21 \\
\hline 12 & H.Chen & University of Arizona, USA & 22 & 1189 & 54.05 & 5 & 6 & 27.27 & 14.41 \\
\hline 13 & J.Kepner & MIT, USA & 22 & 178 & 8.09 & 8 & 1 & 4.55 & 2.16 \\
\hline 14 & W.Dou & Nanjing University, China & 22 & 136 & 6.18 & 9 & 15 & 68.18 & 1.65 \\
\hline 15 & Y.Chang & Leeds Beckett University, U.K. & 22 & 244 & 11.09 & 7 & 12 & 54.55 & 2.96 \\
\hline 16 & F.Xhafa & $\begin{array}{l}\text { Universitat Politecnica de Catalunya, } \\
\text { Spain }\end{array}$ & 22 & 159 & 7.23 & 7 & 18 & 81.82 & 1.93 \\
\hline 17 & C.Rong & University of Stavanger, Norway & 22 & 70 & 3.18 & 4 & 12 & 54.55 & 0.85 \\
\hline 18 & G.B. Giannakis & University of Minnesota, USA & 21 & 168 & 8.00 & 7 & 4 & 19.05 & 2.13 \\
\hline 19 & P.S.Yu & $\begin{array}{c}\text { University of Illinois at Chicago, } \\
\text { USA }\end{array}$ & 21 & 55 & 2.62 & 4 & 5 & 23.81 & 0.70 \\
\hline 20 & $\mathrm{Z} . \mathrm{Xu}$ & $\begin{array}{l}\text { Third Research Institute, Ministry of } \\
\text { Public Security, China }\end{array}$ & 21 & 329 & 15.67 & 7 & 2 & 9.52 & 4.18 \\
\hline
\end{tabular}

TP = Total Papers; TC=Total Citations; $\mathrm{CPP}=$ Citations Per Paper; HI=h-index; ICP=International Collaborative Papers 
Table 10. Relative Citation Index of Top 20 Authors in Big Data Research, 2007-16.

\begin{tabular}{|c|c|c|c|c|c|c|c|c|c|}
\hline S.No & $\begin{array}{l}\text { Name of } \\
\text { Author }\end{array}$ & Affiliation of the Author & $\mathbf{T P}$ & TC & CPP & HI & ICP & $\% \mathrm{ICP}$ & RCI \\
\hline 1 & H.Chen & University of Arizona, USA & 22 & 1189 & 54.05 & 5 & 6 & 27.27 & 14.41 \\
\hline 2 & X.Meng & University of Politehnica of Bucharest, Romania & 14 & 280 & 20.00 & 5 & 3 & 21.43 & 5.33 \\
\hline 3 & F.Herrera & University of Granada, Spain & 23 & 449 & 19.52 & 10 & 14 & 60.87 & 5.21 \\
\hline 4 & J.Wan & South China University of Technology, China & 15 & 290 & 19.33 & 8 & 6 & 40.00 & 5.16 \\
\hline 5 & C.Yang & Universitat Politecnica de Catalunya, Spain & 15 & 290 & 19.33 & 10 & 18 & 120.00 & 5.16 \\
\hline 6 & A.V.Vasilakos & Lulea University of Technology, Sweden & 15 & 279 & 18.60 & 10 & 15 & 100.00 & 4.96 \\
\hline 7 & C.Liu & Universitat Politecnica de Catalunya, Spain & 18 & 325 & 18.06 & 11 & 12 & 66.67 & 4.81 \\
\hline 8 & T.Rabl & University of Toronto, Canada & 20 & 342 & 17.10 & 7 & 18 & 90.00 & 4.56 \\
\hline 9 & L.Wang & Institute of Remote Sensing \& Digital Earth, China & 23 & 380 & 16.52 & 13 & 18 & 78.26 & 4.41 \\
\hline 10 & A.Y.Zomaya & University of Sydney, Australia & 27 & 437 & 16.19 & 11 & 30 & 111.11 & 4.32 \\
\hline 11 & X.Zhang & Pace University, USA & 25 & 394 & 15.76 & 12 & 17 & 68.00 & 4.20 \\
\hline 12 & $\mathrm{Z} . \mathrm{Xu}$ & $\begin{array}{c}\text { Third Research Institute, Ministry of Public Security, } \\
\text { China }\end{array}$ & 21 & 329 & 15.67 & 7 & 2 & 9.52 & 4.18 \\
\hline 13 & V.Markl & Technical University of Berlin, Germany & 16 & 247 & 15.44 & 7 & 4 & 25.00 & 4.12 \\
\hline 14 & R.Ranjan & $\begin{array}{l}\text { Commonwealth Scientific \& Industrial Organization, } \\
\text { Australia }\end{array}$ & 41 & 628 & 15.32 & 15 & 31 & 75.61 & 4.08 \\
\hline 15 & M.Poess & Oracle Corporation, USA & 15 & 229 & 15.27 & 6 & 11 & 73.33 & 4.07 \\
\hline 16 & R.Buyya & University of Melbourne, Australia & 14 & 209 & 14.93 & 5 & 7 & 50.00 & 3.98 \\
\hline 17 & M.J. Carey & University of California, Irvine, USA & 16 & 229 & 14.31 & 8 & 2 & 12.50 & 3.82 \\
\hline 18 & J.Chen & University of Technology Sydney, Australia & 34 & 473 & 13.91 & 12 & 23 & 67.65 & 3.71 \\
\hline 19 & S.Dustdar & Vienna University of Technology, Austria & 15 & 189 & 12.60 & 5 & 11 & 73.33 & 3.36 \\
\hline 20 & S.Wang & Renmin University of China & 15 & 177 & 11.80 & 5 & 1 & 6.67 & 3.15 \\
\hline
\end{tabular}

Top 100 authors in big data research post consistent fall in their productivity and their citation impact as their ranking order drops 1 to 100. This data reveals that top 10 organizations lead in publications productivity and citation impact (Figure 5).

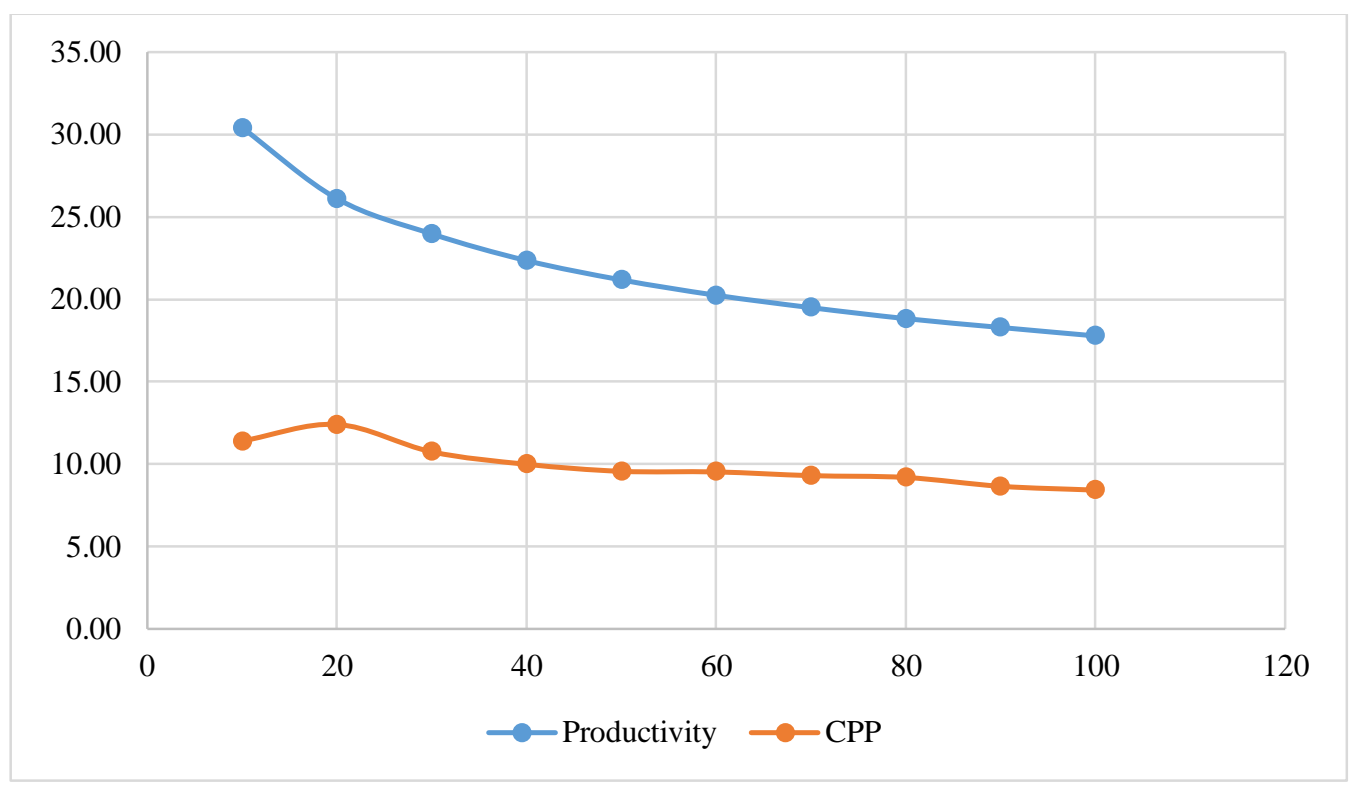

Figure 5. Top 100 Authors in Big Data Research: Quantity vs Quality Performance.

\section{3-9- Medium of Communication in Research}

A total of 1603 journals reported 7538 papers in big data research in 10 years during 2007-16, of which 1025 journals contributed 1-5 papers each, 426 journals 6-10 papers each, 109 journals 11-20 papers each, 18 journals 21-30 papers each, 24 journals 31-100 papers each and 1 journal 135 papers. Of the 1603 reporting journals, 25 accounted for $16.61 \%$ share of 7538 journal papers, each reporting 31 to 135 papers during the period. The top most productive journal (with 135 papers) was Big Data, followed by International Journal of Applied Engineering Research (83 papers), Jisuanji 
Yanjiu Fazhan Computer Research \& Development (72 papers), Future Generation Computer Systems (71 papers), IEEE Access (64 papers), etc. (Table 11).

Table 11. Top 25 Most Productive Journals in Global Big Data Research during 2007-16.

\begin{tabular}{|c|c|c|}
\hline S.No & Name of the Journal & $\begin{array}{c}\text { Number of } \\
\text { Papers }\end{array}$ \\
\hline 1 & Big Data & 135 \\
\hline 2 & International Journal of Applied Engineering Research & 83 \\
\hline 3 & Jisuanji Yanjiu Fazhan Computer Research \& Development & 72 \\
\hline 4 & Future Generation Computer Systems & 71 \\
\hline 5 & IEEE Access & 64 \\
\hline 6 & Journal of Big Data & 59 \\
\hline 7 & Jisuanji Xuebao Chinese Journal of Computers & 51 \\
\hline 8 & Indian Journal of Science \& Technology & 50 \\
\hline 9 & Neurocomputing & 49 \\
\hline 10 & Big Data Research & 48 \\
\hline 11 & Journal of Supercomputing & 48 \\
\hline 12 & Concurrency Computation & 47 \\
\hline 13 & Cluster Computing & 44 \\
\hline 14 & IT Professional & 43 \\
\hline 15 & Ruan Jian Xe Bao Journal of Software & 43 \\
\hline 16 & International Journal of Control Theory \& Applications & 40 \\
\hline 17 & IEEE Network & 36 \\
\hline 18 & NEC Technical Journal & 36 \\
\hline 19 & International Journal of Distributed Sensor Networks & 35 \\
\hline 20 & $\begin{array}{l}\text { Zhongguo Dianji Gongcheng Xuebao Proc. Of the Chinese } \\
\text { Society of Electrical Engineering }\end{array}$ & 35 \\
\hline 21 & $\begin{array}{c}\text { International Journal of Software Engineering \& its } \\
\text { Applications }\end{array}$ & 34 \\
\hline 22 & IEEE Intelligent Systems & 33 \\
\hline 23 & Nature & 33 \\
\hline 24 & International Journal of Pharmacy \& Technology & 32 \\
\hline \multirow[t]{4}{*}{25} & IEEE Internet Computing & 31 \\
\hline & Total of 25 journals & 1252 \\
\hline & Total of the journal world output & 7538 \\
\hline & Share of 25 Journals in journal global output & 16.61 \\
\hline
\end{tabular}

\section{3-10- Highly Cited Papers}

Out of 11104 papers in big data research, less than 1 percent $(96,0.86 \%)$ received 100 to $100+$ citations per paper in 10 years covering the period 2007-16. These 96 highly cited papers together cumulated 21259 citations, with an average of 221.45 citations per paper. Amongst 96 highly cited papers, 64 received 100-200 citations each, 14 received 200-299 citations each, 7 received 300-399 citations each, received 400-599 citations each, and the rest 8 papers were in 520-1098 citation range. These 96 highly cited papers originated from 19 countries. The USA accounted for the highest number of papers (64), followed by China (16 papers), U.K. (11 papers), Canada (6 papers), Australia and Singapore (5 papers each), South Korea (4 papers), Germany, Ireland, Italy and France (3 papers each), Japan and Spain (2 papers), Hong Kong, India, Malaysia, Macau, Netherlands and Turkey (1 papers each).

These 96 highly cited papers involved the participation of 397 authors from 269 organizations. Top organizations which contributed 96 highly cited papers include: University of California, Berkeley, USA, MIT, USA and Nayang Technological University, Singapore (5 papers each), Harvard University, USA (4 papers), University of Cincinnati, $\mathrm{OH}$, USA, Institute of Computing Technology, CAS, China and Tsinghua University, China, University of Toronto, Canada, University of Macau and Imperial College London, U.K. (3 papers each), University of Arizona, USA, Florida Atlantic University, USA. Cornell University, Ithaca, USA, Auburn University, USA, Duke University, USA, University of Southern California, USA, University of Pittsburg, USA, University of Michigan, USA, University of California, San Diego, USA, John Hopkins University, USA, University of Science \& Technology of China, China, Microsoft Research Asia, China, University of Melbourne, Australia, University of Wollongong, Australia and University College London, U.K. (2 papers each), etc. 
These 96 highly cited papers were published in 60 journals, with 3 papers each in Communications of the ACM, IEEE Access and Nature, 2 papers each in Big Data, Dialogue in Human Geography, Harvard Business Review, IEEE Communication Survey \& Tutorials, IEEE Intelligent Systems, IEEE Signal Processing Magazine, IEEE Transactions on Emerging Topics in Computing, International Journal of Production Economics, Journal of Parallel \& Distributed Computing and MIT Sloan Management Review, and 1 paper each in 31 other journals.

\section{4- Conclusion}

The study provides a comprehensive description and analysis of big data research on a series on bibliometric indicators, covering research publications published across the world in 10-year during 2007-16. Big data research cumulated 26566 publications, and averaged 3.75 citations per paper since publication during the period. In addition, the study reports publication trends in big data research by top countries, top institutions, top authors, top journals, and popular subject areas. The study also characterized analytical outcomes on indicators like average citations per paper, relative citation index, average productivity, and country-level international collaboration share.

The study concludes that big data is a subject of recent origin. Given its major potential to impact business, governance, society, healthcare, industry and many other sectors, big data has emerged as a major discipline. Within a decade big data has witnessed big surge in its research growth to $135.6 \%$. Top countries like USA, China, India, UK, and Germany have played a prominent role in the growth of big data research even as 61 countries in all had participated and contributed to research in the field during the period. Top countries, top organizations, and top authors, however, differ in terms of qualitative dimensions in big data research measured on relative citation index, citations per paper, and high citations per paper count. Highly cited papers output is limited to less than 1 percent $(96,0.86 \%)$ of total big data research output in the world. Besides, highly cited papers output is localized to select few countries like USA and China. USA and China are the global leaders in big data research, whereas other high productivity countries in this field are still distant cousins.

\section{5- References}

[1] Big data in action: definition, value, evolutions, benefits and context. https://www.i-scoop.eu/big-data-action-value-context

[2] What is Big Data Analytics? https://www.ibm.com/analytics/hadoop/big-data-analytics\#324371

[3] Moorthy, M., Baby, R. \& Senthamaraiselvi, S (2014). An Analysis for Big Data and its Technologies. International Journal of Computer Science Engineering and Technology (IJCSET), Vol 4, Issue 12, 412-418.

[4] Halevi, Gali and Moed, Henk F. (2012). The Evolution of Big Data as a Research and Scientific Topic: Overview of the Literature. Research Trends (Special Issue), 3-6.

[5] Singh, V.K., Banshal, S.K., Singhal, K. et al. Scientometric mapping of research on 'Big Data'. Scientometrics (2015) 105: 727 741. https://doi.org/10.1007/s11192-015-1729-9.

[6] Singh, Punit Kumar and Singh, Ajay P. Diffusion of Big Data in Indian Scientific Literature: Study of Research Productivity and Scientific Collaboration. Library Philosophy and Practice (e-journal) (2017).

[7] Mathisen, B.M., Roman, D., \& Wienhofen, L.W. (2015). Empirical Big Data Research: A Systematic Literature Mapping. Information Systems arXiv:1509.03045 [cs.DL]

[8] Porter, A.L. Hunag, Y, Schuehle, Y and Youtie, Jan. Meta data: Big data research evolving across disciplines, players, and topics. Conference Paper. June 2015 DOI: 10.1109/BigDataCongress.2015.44. ieeexplore.ieee.org

[9] Mathisen, B.M., Roman, D., \& Wienhofen, L.W. (2015). Empirical Big Data Research: A Systematic Literature Mapping. Information Systems arXiv:1509.03045 [cs.DL]

[10] Kalantari, A., Kamsin, A., Kamaruddin, H.S. et al. (2017). A bibliometric approach to tracking big data research trends. J Big Data (2017) 4: 30. https://doi.org/10.1186/s40537-017-0088-1

[11] Liao, Huchang, Ming Tang, Li Luo, Chunyang Li, Francisco Chiclana, and Xiao-Jun Zeng. "A Bibliometric Analysis and Visualization of Medical Big Data Research.” Sustainability 10, no. 2 (January 11, 2018): 166. doi:10.3390/su10010166.

[12] Gua, Dongxiao, Lia, Jingjing, Lia, Xingguo, Lianga, Changyong., 2017, Visualizing the knowledge structure and evolution of big data research in healthcare informatics. International Journal of Medical Informatics February 2017, 98, 22-32. https://www.sciencedirect.com/science/article/pii/S1386505616302556

[13] Youtie, Jan, Porter, Alan L and Huang, Ying. 2017, Early social science research about big data. Science and Public Policy 1 February 2017, 44(1), 65-74. https://doi.org/10.1093/scipol/scw021. 